Orthopäde 2018 $47: 367$

https://doi.org/10.1007/s00132-018-3559-y

Online publiziert: 19. März 2018

(c) Springer Medizin Verlag GmbH, ein Teil von Springer Nature 2018

CrossMark
Olaf Lorbach

Sporthopaedicum Berlin, Berlin, Deutschland

\section{Aktuelle konservative und operative Konzepte zur Therapie der Omarthrose}

\author{
Update 2018
}

Liebe Kolleginnen und Kollegen,

Das vorliegende Themenheft gibt ein umfassendes Update über die aktuellen Innovationen und Konzepte der konservativen und operativen Behandlung der Omarthrose. Immer noch bekommen Patienten viel zu spät oder gar nicht die richtigen Behandlungsoptionen im Rahmen der Omarthrose aufgezeigt, da gerade die endoprothetische Versorgung im Schultergelenk von Kollegen, die auf diesem Gebiet weniger erfahren sind, immer noch mit großer Skepsis betrachtet wird.

Neben der richtigen Indikationsstellung und der Erfahrung des Operateurs spielt aber auch der richtige Zeitpunkt der Operation eine wichtige Rolle in Bezug auf die zu erwartende Schulterfunktion. In den letzten 50 Jahren ist die Schulterendoprothetik stetig weiterentwickelt worden, sodass hier mittlerweile neben guten klinischen Ergebnissen auch Standzeiten erreicht werden, welche durchaus mit denen der Knie- und Hüftendoprothetik vergleichbar sind.

Nicht jede initiale Omarthrose muss gleich operiert werden. Daher werden in Detail auch die möglichen konservativen Therapieoptionen aufgezeigt, welche insbesondere in frühen Stadien der Arthrose mit geringer Funktionseinschränkung einen guten Erfolg erreichen können. Sind diese nicht erfolgreich, gibt es - gerade in frühen Stadien der Arthrose - auch vielversprechende gelenkerhaltende operative Therapien, welche zwar die Arthrose nicht direkt adressieren, jedoch über die Behandlung der aus der
Arthrose resultierenden Begleitpathologien, wie Bizepssehnenreizung und Bewegungseinschränkung, nicht selten die Beschwerden auf ein zufriedenstellendes Maß reduzieren können. Gerade beim jungen Patienten lässt sich damit nicht selten die endoprothetische Versorgung zeitlich herauszögern und in bestimmten Fällen sogar vermeiden.

Liegt bereits eine fortgeschrittene Omarthrose mit entsprechender Beschwerdesymptomatik und Funktionsverlust vor, ist der endoprothetische Ersatz häufig die einzige Erfolg versprechende Lösung. Im Detail wird auf die Indikationen, aktuelle Implantate sowie die klinischen Ergebnisse der anatomischen Schulterprothese und der inversen Schulterprothese bei additiver Rotatorenmanschetteninsuffizienz Schulterprothese wird aufgrund der guten klinischen Ergebnisse zunehmend bereits primär bei älteren Patienten mit grenzwertiger Rotatorenmanschettenintegrität oder auch bei komplexen Humeruskopffrakturen eingesetzt, und stellt gerade bei älteren Patienten eine echte Alternative zur Osteosynthese und Frakturprothese dar.

Zwangsläufig führt die steigende Zahl an Endoprothesen aber auch, gerade in diesem Zusammenhang, zu einer signifikanten Zunahme der Revisionsoperationen nach implantierter Schulterprothese, in denen ein klarer Therapiealgorithmus einen wichtigen Faktor für das Gelingen der Operation darstellt. Darüber hinaus bieten aktuelle Implantate mit schaftfreier Versorgung und Modularität der Komeingegangen. Insbesondere die inverse ponenten viele neue Möglichkeiten, die im Revisionsfall den Prothesenwechsel erheblich erleichtern können.

Das Themenheft wird durch die Rehabilitation und Nachbehandlung nach anatomischer und inverser Schulter-TEP abgerundet.

Ich hoffe, dass dieses Themenheft zur Klärung einiger klinisch relevanter Fragen in Bezug auf die Behandlung der glenohumeralen Arthrose unter Berücksichtigung der aktuellen Literatur beitragen kann.

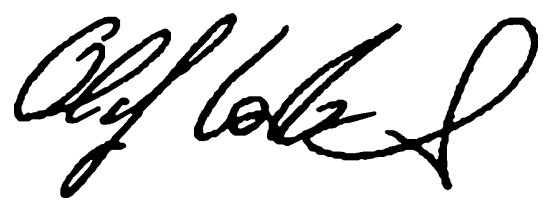

Olaf Lorbach

\section{Korrespondenzadresse}

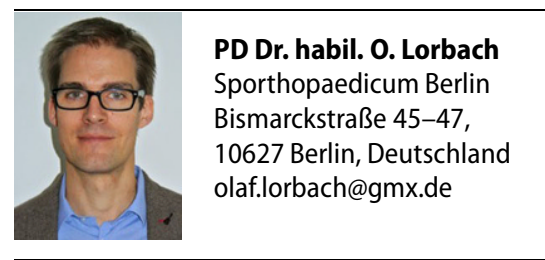

Interessenkonflikt. O. Lorbach gibt an, dass kein Interessenkonflikt besteht. 